\title{
Assessment of RNA carrier function in peptide amphiphiles derived from the HIV fusion peptide
}

\author{
Yaowalak Pratumyot • Oscar Torres • Dennis Bong*
}

Department of Chemistry and Biochemistry, The Ohio State University, 100 W. 18th Avenue, Columbus, OH 43210.

*Corresponding author email: bong.6@osu.edu 


\begin{abstract}
A small library of amphiphilic peptides has been evaluated for duplex RNA carrier function into A549 cells. We studied peptides in which a $C$-terminal 7-residue cationic domain is attached to a neutral/hydrophobic 23residue domain that is based on the viral fusion peptide of HIV. We also examined peptides in which the cationic charge was evenly distributed throughout the peptide. Strikingly, subtle sequence variations in the hydrophobic domain that do not alter net hydrophobicity result in wide variation in RNA uptake. Additionally, cyclic cystine variants are much less active as RNA carriers than their open-chain cysteine analogs. With regard to electrostatic effects, we find that lysine is less effective than arginine in facilitating uptake, and that even distribution of cationic residues throughout the peptide sequence results in especially effective RNA carrier function. Overall, minor changes in peptide hydrophobicity, flexibility and charge distribution can significantly alter carrier function. We hypothesize this is due to altered properties of the peptide-RNA assembly rather than peptide secondary structure.
\end{abstract}

Keywords: carrier, amphiphilic peptide, electrostatics, transport, nucleic acids

\title{
Introduction
}

Synthetic and natively derived peptide carriers for nucleic acid delivery have been the subject of many studies [1,2]. The relationship between membrane activity of peptides against synthetic lipid vesicles [3-5] and biomembrane activity remains unpredictable [6-8]. We have previously reported on the synthetic vesicle membrane fusion [9-14] and lysis activity of a library of HIV fusion peptide variants [15]. One major goal of this prior study was to understand the origins of membrane activity within the framework of a viral fusion peptide. To probe the biophysical function of the monomeric form of these hydrophobic peptides, each variant of the 23-residue fusion peptide domain was synthesized with a $C$-terminal cationic heptapeptide to promote solubility [16,17]. Interestingly, we found considerable variation in membrane activity upon shuffling the amino acid sequence of the HIV fusion peptide; moreover, within this 30-residue diblock peptide amphiphile architecture it was possible to identify variants that were purely fusogenic or lytic against negatively charged synthetic lipid vesicle membranes [15]. Though derived from native viral sequences, the separation of hydrophobic and polar residues into two distinct, fused peptide domains is more akin to polymer carriers [18-20] than native transduction domains [21-23]. Herein, we describe our studies on the function of these peptide amphiphiles as carriers for duplex RNA cargo across cell membranes. The cationic domain of each peptide, previously used to facilitate binding to anionic membranes [15], was used in this study to electrostatically bind fluorescein-labeled $21 \mathrm{nt}$ duplex RNA (Figure 1). We found that the peptide-RNA complexes could be taken up into cells in

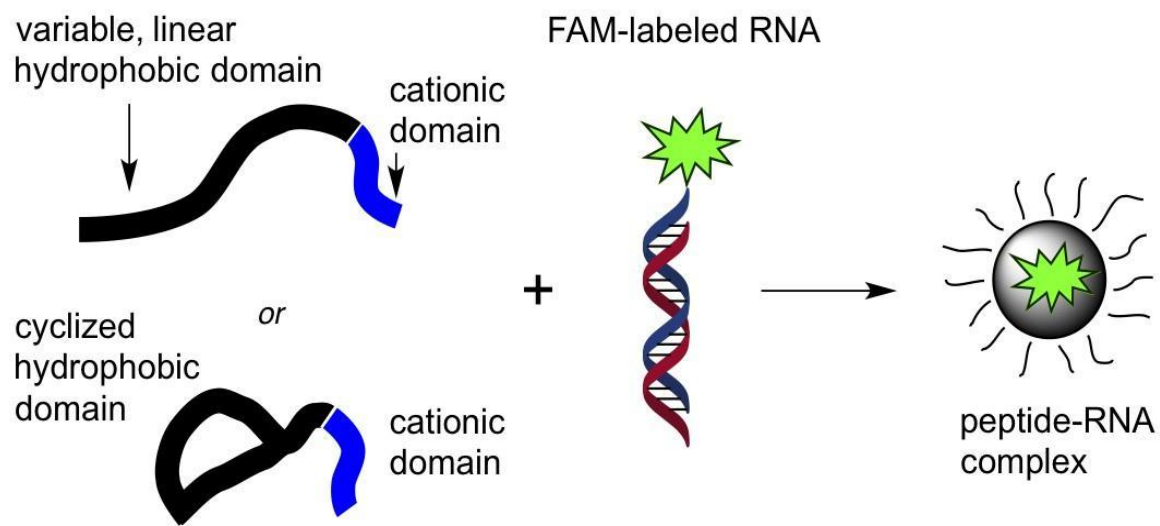

Figure 1. Illustration of peptide diblock architecture and electrostatic complexation with fluorescein-labeled duplex RNA. 
culture without additional transfection agent, as judged by FACS. However, it was much more difficult to determine a clear relationship between sequence and function. Sequences that displayed strong fusogenic membrane activity against synthetic vesicles were not necessarily effective RNA transporters into A549 cells in culture; indeed, no clear correlation could be found between vesicle data and cell uptake data (Table 1). Furthermore, while a strongly lytic peptide with evenly distributed cationic residues was highly effective in RNA transport, a similarly patterned peptide with a different sequence by nearly identical overall hydrophobicity was a totally ineffective RNA carrier. The sequence dependence of peptide-RNA transport across biomembranes has proven to be considerably more difficult than examination of membrane activity of synthetic vesicles. Generally, uptake is indeed strongly sensitive to sequence variation and depends less on overall

\section{Table 1. Peptide sequences and uptake activity}

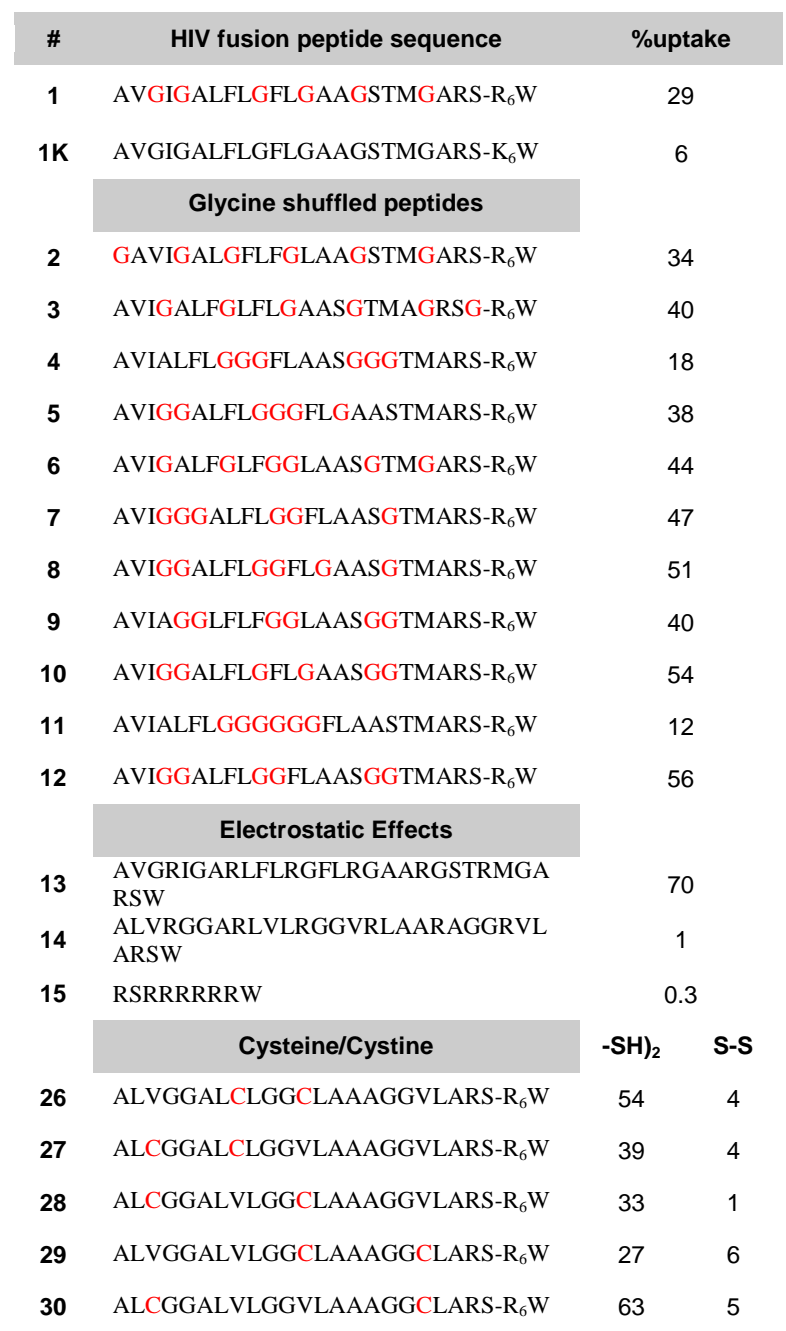

hydrophobicity. Despite the poor correlation between synthetic membrane activity and RNA cellular delivery, we find broad guidelines for improving carrier function in the peptide diblock amphiphile architecture.

\section{Materials and Methods}

General. All siRNA and 5X siRNA buffer were purchased from Thermo Scientific (Dharmacon). Phenol Red-free DMEM, FBS, Penicillin-Streptomycin, 0.05\% Trypsin-EDTA (1X), 1X DPBS, Hoechst 33258 and Opti-Mem were purchased from Invitrogen. Heparin sodium salt (from porcine intestinal mucosa) and Fluoroshield ${ }^{\mathrm{TM}}$ (mounting medium) were purchased from Sigma Aldrich. 
Peptide Synthesis. Peptides were synthesized using standard Fmoc solid phase peptide chemistry (Rink LS resin) on an Apex 396 AAPPTec peptide synthesizer and purified to homogeneity by RP-HPLC, as described previously [15]. Briefly, diisopropyl carbodiimide (DIC) / HOBt was used as a coupling reagent with 1-hour standard coupling time. Peptides were cleaved from resin using a mixture of TFA: thioanisole: m-cresol: ethanedithiol: $\mathrm{H}_{2} \mathrm{O}$ in the ratio of 87.5:5:2.5:2.5:2.5 for 3.5 hours. Peptides were precipitated from the cleavage cocktail with cold diethyl ether and purified by reversed-phase HPLC $\left(\mathrm{C}_{18}\right)$ on water-acetonitrile $(0.1 \%$ TFA) gradients. Stock solutions of peptides were prepared in deionized $\mathrm{H}_{2} \mathrm{O}$ and stored at $-20^{\circ} \mathrm{C}$. The concentration of peptides were determined by the UVabsorbance (Trp) at $280 \mathrm{~nm}\left(\varepsilon=5502 \mathrm{M}^{-1} \mathrm{~cm}^{-1}\right)$.

Nucleic acids. Duplex FAM-labeled RNA was purchased from Dharmacon: 5'-(FAM)-CUUACGCUGAGUACUUCGA-dTdT-3' and 5'-UCGAAGUACUCAGCGUAAG-dTdT-3'.

RNA uptake. A549 cells were seeded in 12 well plates at 150,000 cells/well and incubated in growth medium containing 90\% phenol red-free DMEM, 10\% RBS, 100 units $/ \mathrm{ml}$ penicillin, $100 \mu \mathrm{g} / \mathrm{ml}$ streptomycin at $37{ }^{\circ} \mathrm{C}$, and $5 \% \mathrm{CO}_{2}$ and grown for $24 \mathrm{hr}$. Prior to transfection, media was removed and $1 \mathrm{ml}$ of fresh DMEM added to each well. Cells were then transfected with 10, 20, and 40 pmol FAM-GL3 siRNA duplex complexed with peptides at N/P (Nitrogen-based cation to Phosphorous-based anion) ratios of 1-15 to determine the optimal condition for transfection. At RNA concentrations $8 \mathrm{nM}$ and $17 \mathrm{nM}$, the transfection efficiency was low (less than $10 \%$ positive cells) and could not be improved by increasing N/P ratio. A significant increase in uptake was observed at $33 \mathrm{nM}$ RNA, improved by increasing N/P ratio from 5-9, with maximum uptake at $\mathrm{N} / \mathrm{P}=9$ and decreasing efficiency at $\mathrm{N} / \mathrm{P}>9$. Uptake experiments were thus performed with $\mathrm{N} / \mathrm{P}=9$ at $33 \mathrm{nM}$ total RNA.

Transfections were performed in triplicate. RNA/peptide complexes were prepared by incubation of $120 \mu$ of peptide in Opti-Mem with $120 \mu \mathrm{l}$ of 12, 24 or 48 pmol RNA for $1 \mathrm{hr} .200 \mu \mathrm{l}$ of the RNA/peptide complex solution was added to cells in each well and incubated $6 \mathrm{hr}$ at $37^{\circ} \mathrm{C}, 5 \% \mathrm{CO}_{2}$. The medium was then removed, and cells were incubated in fresh media for another $6 \mathrm{hr}$ at $37{ }^{\circ} \mathrm{C}, 5 \% \mathrm{CO}_{2}$. Cells were then washed with DPBS $(3 \mathrm{x})$ and heparin solution (15 units $/ \mathrm{ml}, 3 \mathrm{x}$ ) three times Cells were incubated with heparin solution for $20 \mathrm{~min}$ at $37{ }^{\circ} \mathrm{C}, 5 \% \mathrm{CO}_{2}$ for each wash. After heparin treatment, cells were again washed with 1X DPBS and trypsinized $\left(37^{\circ} \mathrm{C}, 10-15\right.$ min), resuspended in $700 \mu \mathrm{L}$ media and spun down and washed with DPBS. Cells were resuspended and fixed in $4 \%$ paraformaldehyde $\left(4^{\circ} \mathrm{C}, 10 \mathrm{~min}\right)$, washed in DPBS $(2 \mathrm{x})$ and analyzed by a LSR-II flow cytometer gated on FAM fluorescence to determine \%positive cells on volume of 10,000 cells.

\section{Results and Discussion}

A small peptide library was prepared using solid phase peptide synthesis as described previously [15] and examined for RNA carrier function. Each peptide was complexed with fluorescein-labeled $21 \mathrm{nt}$ duplex RNA. Like known RNA carriers such as lipofectamine, the peptide-RNA complexes underwent higher order assembly into a sample of particles of heterogeneous distribution, as judged by light scattering. The polydispersity of the particle assembly precludes reliable assessment of particle size, though they appear to be in the range of hundreds of nanometers to microns. RNA carrier function against A549 cells was found to vary significantly with sequence, with no clear functional relationship between sequence and function, though general trends could be observed. This is perhaps not surprising, given the greater complexity of a biomembrane relative to a synthetic vesicle and the variability scattering sizes found in RNA-peptide complexation.

A subset of 12 peptides with distinct hydrophobic and cationic domains was studied wherein the hydrophobic domains had amino acid compositions identical to that of the 23-residue HIV fusion peptide. This viral fusion domain has 6 glycines, which are thought to be essential to viral infectivity [24,25]. To probe the importance of these residues on RNA transport, the glycines were "shuffled" through the hydrophobic domain while the order of the nonglycine residues was held constant. Analysis of this glycine-shuffled library indicated that placement of the glycines in the hydrophobic domain strongly affects RNA uptake. Indeed, a wide range of uptake activity is observed for these peptides that have identical hydrophobicity. On comparison of the two most effective carriers $(\mathbf{1 0} \& \mathbf{1 2})$ with the two least effective $(\mathbf{4} \& \mathbf{1 1})$, it appears that the major difference in sequence is between residues 4-9. Differential glycine placement shifts a hydrophobic cluster towards the $C$-terminus in $10 \& 12$ and towards the $N$-terminus in 4 \& 11 (Figure 2). The subtle $\mathrm{N}$-terminal shift in hydrophobicity results in a significant ( 40\%) drop in uptake function for peptides 4 \& 11. 


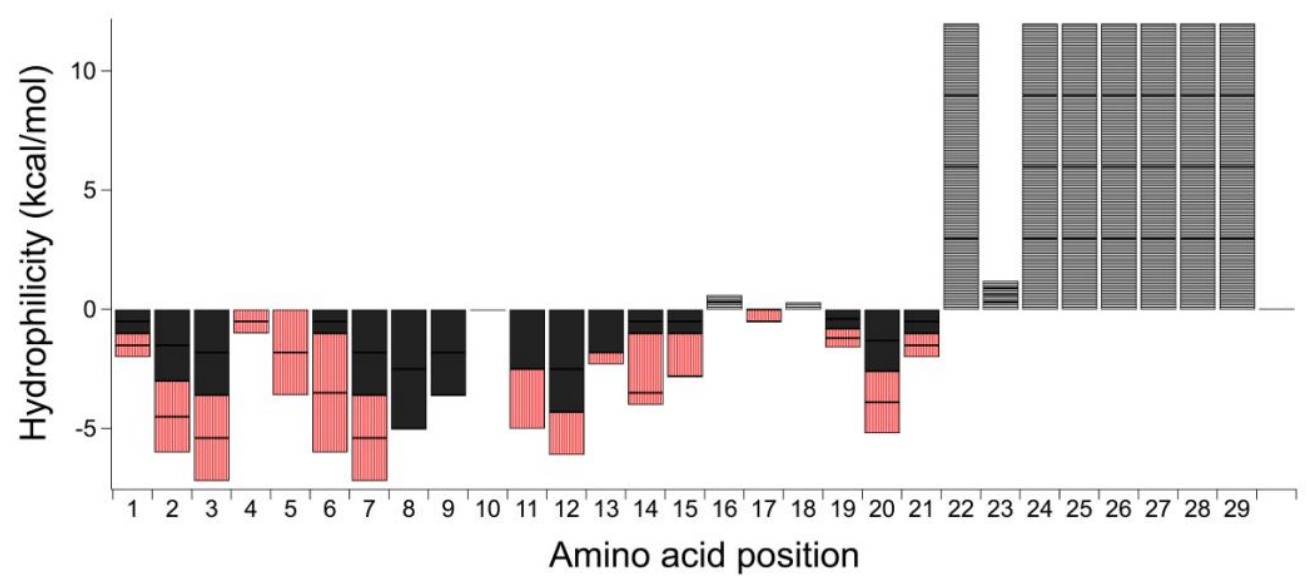

Figure 2. Stacked hydrophilicity plots as a function of amino acid position $(N$-terminus $=1)$ for glycine shuffled peptides $\mathbf{4}, \mathbf{1 0}$, $11 \& 12$. Hydrophobic residues in peptides $\mathbf{4}$ and $\mathbf{1 1}$ are shown as red hashed bars (<18\% RNA positive cells) and as black bars in 10 and 12 (>50\% RNA positive cells). Hydrophilic residues have positive values and are shown as black hashed bars for all peptides.

We next investigated the effect of the cationic domain on RNA uptake. We found that arginine was much more effective than lysine in facilitating uptake, labeling $23 \%$ more of the cell population. Indeed, it is well known that guanidinium groups have a greater functional role in membrane activity relative to ammonium groups [23,26]. Less predictable was the effect of charge distribution along the peptide backbone. A heptaarginine peptide alone was not an effective RNA carrier $(0.3 \%$ positive cells). For cellular uptake, a peptide carrier with an even distribution of 7 arginines along the backbone appears to be significantly more potent than peptides with separated hydrophobic and cationic domains. Peptide 13, comprised of the HIV viral fusion sequence punctuated with 7 arginine residues distributed along its 30 residue length, yielded the highest RNA uptake of 70\%. This charge pattern is more closely associated with lytic antimicrobial peptides than fusogenic or transport peptides [6,27]. However, peptide 14, which has very similar hydrophobicity and the identical spacing of charged groups, is essentially not effective as an RNA carrier. In contrast to the efficient carrier activity of $13(70 \%)$, peptide 14 was only able to increase fluorescence of $\sim 1 \%$ of the cell population. Thus, information critical to RNA transport function is encoded within the primary sequence of $\mathbf{1 3}$ that is more important than overall hydrophobicity and charge patterning. Comparison of the pattern of hydrophobicity [28,29] in $\mathbf{1 3} \& \mathbf{1 4}$ suggests the transport active $\mathbf{1 3}$ possesses hydrophobic residues near the center of the sequence that are absent in 14, though the differences are subtle (Figure 3). This is similar to our finding with the glycine shuffled library.

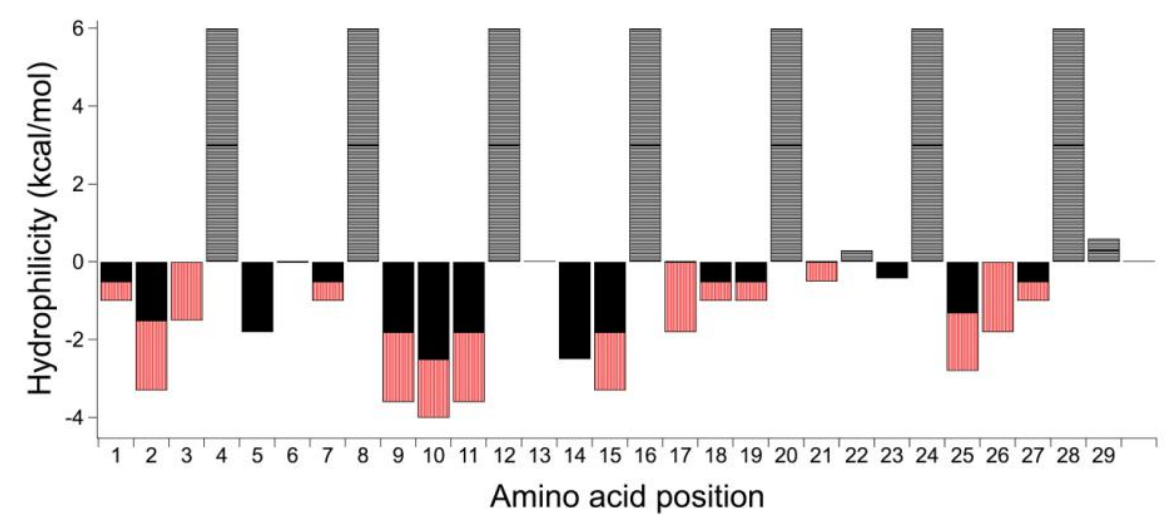

Figure 3. Stacked hydrophilicity plots as a function of amino acid position $(N$-terminus $=1)$ for charge distributed peptides $\mathbf{1 3} \&$ 14. Hydrophobic residues in peptide 14 ( 1\% RNA positive cells) are shown as red hashed bars and as black bars in 13 ( $70 \%$ RNA positive cells). Hydrophilic residues have positive values and are shown as black hashed bars for both peptides.

Shuffling the placement of glycine residues along the peptide backbone has consequences for the placement of hydrophobic groups, as well as for overall peptide flexibility. Furthermore, it appears that both sequence and overall 
hydrophobic content can affect uptake, suggesting the importance of steric accessibility to the membrane. To broadly assess the impact of peptide flexibility, we prepared a series of peptides with identical hydrophobic content (Table 1) that contained two cysteine residues in the hydrophobic domain, located at 5 sets of locations. Upon thiol oxidation, these peptides formed cyclic peptide cystines with variation in the size and location of the peptide macrocycle. There was again variation in the RNA carrier properties due to cysteine placement; throughout the peptide, Cys was used to substitute for $\mathrm{Val}$; though they are similar in steric demand, they are not identical in hydrophobicity. However, in all cases, oxidative cyclization resulted in a precipitous drop in RNA uptake, in one case reducing uptake from $63 \%$ to $5 \%$ (Figure 4). These data indicate the importance of a flexible, linear hydrophobic domain in the diblock peptide carrier design.

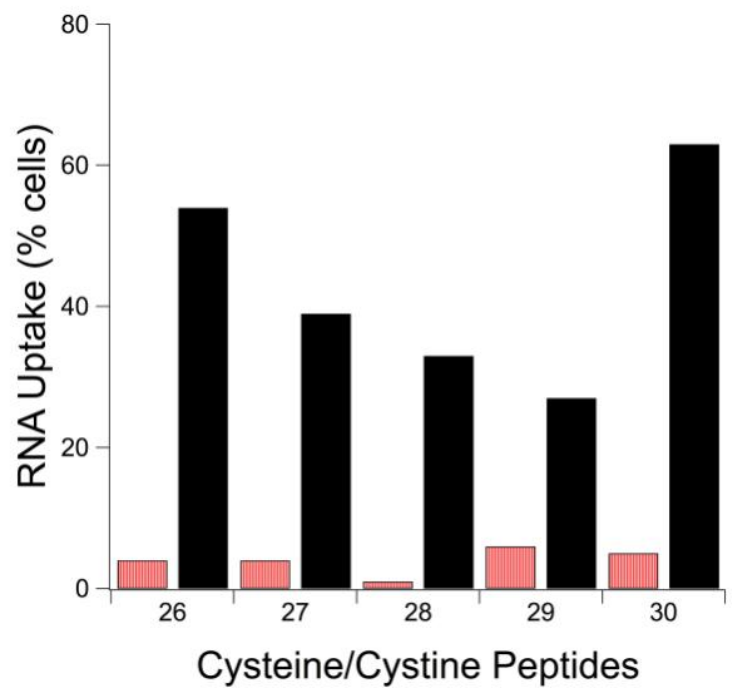

Figure 4. RNA uptake (\% positive cells) mediated by peptide carriers $\mathbf{2 6}$ to $\mathbf{3 0}$. Oxidized (cystine) peptides are indicated as red hashed bars while reduced (dicysteine) peptides are indicated as black bars.

\section{Conclusions}

Taken together, these experiments reveal wide variability in RNA carrier function within a set of highly similar cationic peptide amphiphiles. Subtle variation in the sequences was found to strongly affect cell uptake. Indeed, not just overall hydrophobicity, but placement of hydrophobic clusters is important, with increased uptake when the hydrophobic domain $\sim 10$ residues from the site of peptide-RNA complexation rather than $\sim 15$ residues away. Furthermore, a cyclic cystine peptide carrier has greatly suppressed carrier activity relative to an open-chain dicysteine peptide. As the cystine and dicysteine peptides have nearly identical hydrophobicity, this dependence of carrier function on thiol oxidation state suggests that steric issues may prevent the cystine-RNA complex from mediating transport. Steric accessibility may generally play an important role in guiding cross membrane transport of RNA-peptide complexes, given the sensitivity of RNA-uptake on the placement of hydrophobic residues in the peptide carrier. Indeed, the carrier efficiency of both diblock amphiphile and charge-distributed peptide designs was strongly dependent on sequence, though the precise relationship between primary structure and function remains elusive. The peptide carriers likely complex the anionic RNA backbone via the cationic residues, and the peptide sidechains drive complex assembly into particles via hydrophobic interactions. We speculate that higher order complex assembly[30] into particles could be also be important in determining membrane binding and cellular uptake [31]. The sensitivity of cellular uptake to changes in carrier sequence is remarkable and will be more clearly defined through synthesis and evaluation in future work.

\section{Acknowledgements}

This work was supported in part by the National Science Foundation (NSF DMR 1410232) and the National Institutes of Health (GM111995-01A1). 


\section{REFERENCES}

[1] T. Endoh, T. Ohtsuki, Cellular siRNA delivery using cell-penetrating peptides modified for endosomal escape, Adv. Drug Deliv. Rev. 61 (2009) 704-709.

[2] F. Simeoni, M.C. Morris, F. Heitz, G. Divita, Insight into the mechanism of the peptide-based gene delivery system MPG: implications for delivery of siRNA into mammalian cells, Nucleic Acids Res. 31 (2003) 2717-2724.

[3] V. Buzon, E. Padros, J. Cladera, Interaction of Fusion Peptides from HIV gp41 with Membranes: A Time-Resolved Membrane Binding, Lipid Mixing, and Structural Study, Biochemistry. 44 (2005) 13354-13364.

[4] D.-K. Chang, S.-F. Cheng, W.-J. Chien, The amino-terminal fusion domain peptide of human immunodeficiency virus type $1 \mathrm{gp} 41$ inserts into the sodium dodecyl sulfate micelle primarily as a helix with a conserved glycine at the micelle-water interface, J. Virol. 71 (1997) 6593-6602.

[5] M.E. Haque, V. Koppaka, P.H. Axelsen, B.R. Lentz, Properties and structures of the influenza and HIV fusion peptides on lipid membranes: Implications for a role in fusion, Biophys. J. 89 (2005) 3183-3194.

[6] P. Joanne, P. Nicolas, C. El Amri, Antimicrobial peptides and viral fusion peptides: how different they are?, Protein Pept. Lett. 16 (2009) 743-750.

[7] D.V. Zhelev, N. Stoicheva, P. Scherrer, D. Needham, Interaction of Synthetic HA2 Influenza Fusion Peptide Analog with Model Membranes, Biophys. J. 81 (2001) 285-304.

[8] K. Weise, J. Reed, Fusion peptides and transmembrane domains of fusion proteins are characterized by different but specific structural properties, Chembiochem. 9 (2008) 934-943.

[9] M. Ma, A. Paredes, D. Bong, Intra- and intermembrane pairwise molecular recognition between synthetic hydrogenbonding phospholipids, J. Am. Chem. Soc. 130 (2008) 14456-14458.

[10] Y. Gong, Y. Luo, D. Bong, Membrane activation: selective vesicle fusion via small molecule recognition, J. Am. Chem. Soc. 128 (2006) 14430-14431.

[11] M. Ma, D. Bong, Controlled Fusion of Synthetic Lipid Membrane Vesicles, Acc. Chem. Res. 46 (2013) 2988-2997.

[12] M. Ma, Y. Gong, D. Bong, Lipid Membrane Adhesion and Fusion Driven by Designed, Minimally Multivalent HydrogenBonding Lipids, J. Am. Chem. Soc. 131 (2009) 16919-16926.

[13] M. Ma, D. Bong, Directed peptide assembly at the lipid-water interface cooperatively enhances membrane binding and activity, Langmuir. 27 (2011) 1480-1486.

[14] Y. Gong, M. Ma, Y. Luo, D. Bong, Functional determinants of a synthetic vesicle fusion system, J. Am. Chem. Soc. 130 (2008) 6196-6205.

[15] O. Torres, D. Bong, Determinants of Membrane Activity from Mutational Analysis of the HIV Fusion Peptide, Biochemistry. 50 (2011) 5195-5207.

[16] R. Yang, M. Prorok, F.J. Castellino, D.P. Weliky, A Trimeric HIV-1 Fusion Peptide Construct Which Does Not SelfAssociate in Aqueous Solution and Which Has 15-Fold Higher Membrane Fusion Rate, J. Am. Chem. Soc. 126 (2004) $14722-14723$.

[17] W. Qiang, D.P. Weliky, HIV Fusion Peptide and Its Cross-Linked Oligomers: Efficient Syntheses, Significance of the Trimer in Fusion Activity, Correlation of beta Strand Conformation with Membrane Cholesterol, and Proximity to Lipid Headgroups, Biochemistry. 48 (2009) 289-301.

[18] J. Nguyen, F.C. Szoka, Nucleic Acid Delivery: The Missing Pieces of the Puzzle?, Acc. Chem. Res. 45 (2012) 1153-1162.

[19] N. Nishiyama, K. Kataoka, Current state, achievements, and future prospects of polymeric micelles as nanocarriers for drug and gene delivery, Pharmacol. Ther. 112 (2006) 630-648.

[20] S. Bandyopadhyay, X. Xia, A. Maiseiyeu, G. Mihai, S. Rajagopalan, D. Bong, Z-Group Ketone Chain Transfer Agents for RAFT Polymer Nanoparticle Modification via Hydrazone Conjugation, Macromolecules. 45 (2012) 6766-6773.

[21] J.S. Wadia, R.V. Stan, S.F. Dowdy, Transducible TAT-HA fusogenic peptide enhances escape of TAT-fusion proteins after lipid raft macropinocytosis, Nat. Med. . 10 (2004) 310-315.

[22] B.R. Meade, S.F. Dowdy, The road to therapeutic RNA interference (RNAi): Tackling the 800 pound siRNA delivery gorilla, Discov. Med. 8 (2009) 253-256.

[23] S. Futaki, T. Suzuki, W. Ohashi, T. Yagami, S. Tanaka, K. Ueda, et al., Arginine-rich peptides An abundant source of membrane-permeable peptides having potential as carriers for intracellular protein delivery, J. Biol. Chem. 276 (2001) 5836-5840.

[24] M.D. Delahunty, I. Rhee, E.O. Freed, J.S. Bonifacino, Mutational analysis of the fusion peptide of the human immunodeficiency virus type 1: identification of critical glycine residues, Virology. 218 (1996) 94-102.

[25] J. Munch, L. Standker, K. Adermann, A. Schulz, M. Schindler, R. Chinnadurai, et al., Discovery and optimization of a natural HIV-1 entry inhibitor targeting the gp41 fusion peptide, Cell. 129 (2007) 263-275.

[26] M. Tang, A.J. Waring, R.I. Lehrer, M. Hong, Effects of guanidinium-phosphate hydrogen bonding on the membrane-bound structure and activity of an arginine-rich membrane peptide from solid-state NMR spectroscopy, Angew. Chem. Int. Ed Engl. 47 (2008) 3202-3205. 
[27] W.C. Wimley, Describing the Mechanism of Antimicrobial Peptide Action with the Interfacial Activity Model, ACS Chem. Biol. 5 (2010) 905-917.

[28] T.P. Hopp, K.R. Woods, Prediction of protein antigenic determinants from amino acid sequences, Proc. Natl. Acad. Sci. U. S. A. 78 (1981) 3824-3828.

[29] J.M. Parker, D. Guo, R.S. Hodges, New hydrophilicity scale derived from high-performance liquid chromatography peptide retention data: correlation of predicted surface residues with antigenicity and X-ray-derived accessible sites, Biochemistry. 25 (1986) 5425-5432.

[30] J. Pathak, K. Rawat, V.K. Aswal, H.B. Bohidar, Interactions in globular proteins with polyampholyte: coacervation route for protein separation, RSC Adv. 5 (2015) 13579-13589.

[31] A. Lesniak, A. Salvati, M.J. Santos-Martinez, M.W. Radomski, K.A. Dawson, C. Åberg, Nanoparticle adhesion to the cell membrane and its effect on nanoparticle uptake efficiency, J. Am. Chem. Soc. 135 (2013) 1438-1444. 
GRAPHICAL ABSTRACT

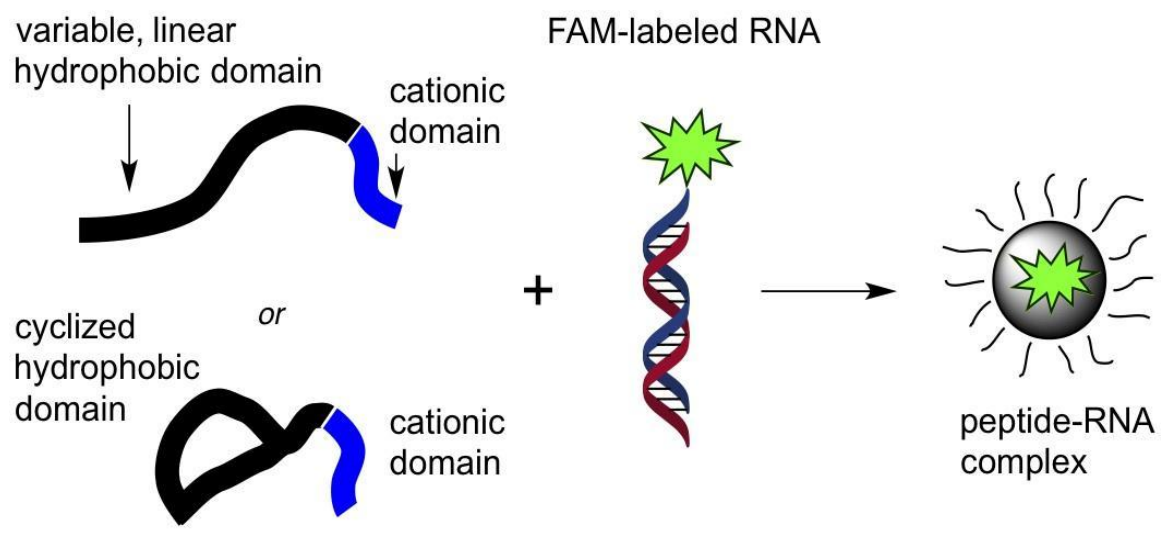

